\title{
17. GRAIN-SIZE AND CARBON/CARBONATE ANALYSES, LEG 39
}

\author{
Brad Scott, Scripps Institution of Oceanography, La Jolla, California
}

\section{GRAIN-SIZE ANALYSIS}

Sand-silt-clay distribution was determined on 10-cc sediment samples collected at the time the cores were split and described. The grain-size analyses results for Leg 39 are listed in Table 1.

Standard sieve and pipette methods were used to determine the grain-size distribution. For detailed stepby-step procedures, see Volume 4 of the Initial Reports of the Deep Sea Drilling Project (Bader, Gerard, et al., 1970). Sampling depths and times utilized in the standard pipette analysis were calculated using equations derived from Stokes settling velocity equation (Krumbein and Pettijohn, 1938, p. 95-96):

$$
\begin{aligned}
\frac{D}{t} & =V=\frac{2 g r^{2}\left(d_{1}-d_{2}\right)}{9 \eta} \\
t & =\frac{9 D \eta}{2 g r^{2}\left(d_{1}-d_{2}\right)}
\end{aligned}
$$

where

$V=$ velocity, in $\mathrm{cm} / \mathrm{sec}$

$t=$ time, in sec*

$D=$ depth pipette is inserted, in $\mathrm{cm}$

$g \quad=$ gravity, in $\mathrm{cm} / \mathrm{sec}^{2 *}$

$r \quad=$ radius of individual particles, in $\mathrm{cm}^{*}$

$d_{1}=$ density of solid particles arbitrarily set at 2.675 $\mathrm{g} / \mathrm{cc}$

$d_{2}=$ absolute density of distilled water at different temperatures (Hodgman et al., 1960, p. 2129)

$\eta \quad=$ viscosity of distilled water in poises at different temperatures (Hodgman et al., 1960, p. 2181)

The sediment classification used in Table 1 is that of Shepard (1954) with the sand, silt, and clay boundaries based on the Wentworth (1922) scale (Figure 1). Note that this classification is applied regardless of sediment type and origin. The sand, silt, and clay fractions are composed of particles whose diameters range from 2000 to $62.5 \mu \mathrm{m}$, and less than $3.91 \mu \mathrm{m}$, respectively.

The reproducibility of the grain-size analysis has been previously tested (Boyce, 1972), and it was found that over a period of time with several operators the reproducibility for sand-silt-clay fractions is $\pm 2.5 \%$ (absolute).

*Five figures were used in calculations to avoid rounding off variations.
TABLE 1

Grain-Size Determination, Leg 39, Site 353

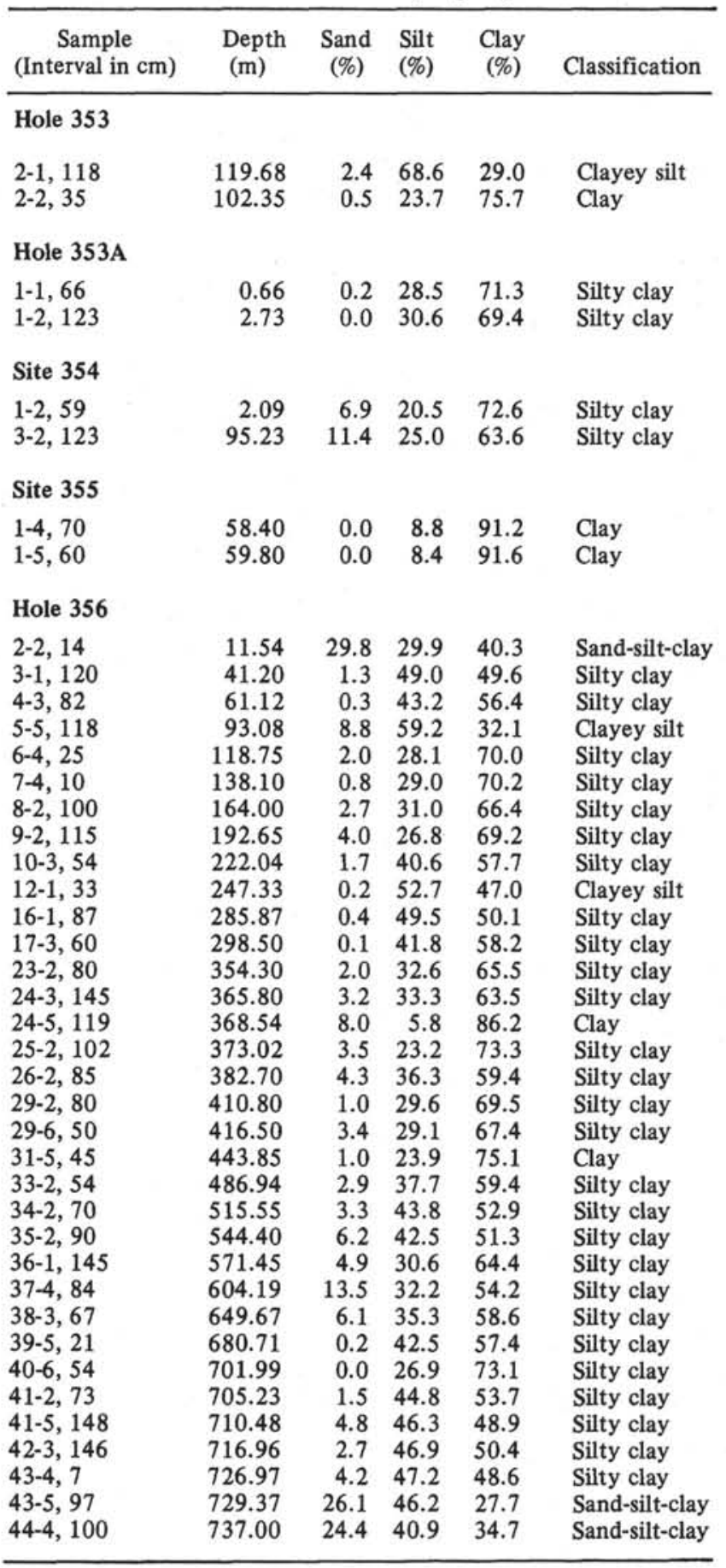


TABLE 1 - Continued

\begin{tabular}{|c|c|c|c|c|c|}
\hline $\begin{array}{c}\text { Sample } \\
\text { (Interval in } \mathrm{cm} \text { ) }\end{array}$ & $\begin{array}{l}\text { Depth } \\
\text { (m) }\end{array}$ & $\begin{array}{c}\text { Sand } \\
(\%)\end{array}$ & $\begin{array}{l}\text { Silt } \\
(\%)\end{array}$ & $\begin{array}{l}\text { Clay } \\
(\%)\end{array}$ & Classification \\
\hline \multicolumn{6}{|l|}{ Hole 356A } \\
\hline $\begin{array}{l}1-4,88 \\
1-5,100 \\
2-5,19\end{array}$ & $\begin{array}{l}24.73 \\
26.35 \\
34.69\end{array}$ & $\begin{array}{l}0.1 \\
0.7 \\
0.9\end{array}$ & $\begin{array}{l}39.4 \\
46.0 \\
40.1\end{array}$ & $\begin{array}{l}60.6 \\
53.3 \\
59.0\end{array}$ & $\begin{array}{l}\text { Silty clay } \\
\text { Silty clay } \\
\text { Silty clay }\end{array}$ \\
\hline \multicolumn{6}{|l|}{ Site 357} \\
\hline $\begin{array}{l}1-2,118 \\
6-5,61 \\
43.6,61\end{array}$ & $\begin{array}{r}2.68 \\
53.11 \\
729.54\end{array}$ & $\begin{array}{l}62.9 \\
17.5 \\
10.2\end{array}$ & $\begin{array}{l}16.2 \\
47.8 \\
35.6\end{array}$ & $\begin{array}{l}20.9 \\
34.7 \\
54.2\end{array}$ & $\begin{array}{l}\text { Clayey sand } \\
\text { Clayey silt } \\
\text { Silty clay }\end{array}$ \\
\hline \multicolumn{6}{|l|}{ Site 358} \\
\hline $\begin{array}{l}1-4,53 \\
5-1,19 \\
6-3,97 \\
7-2,13 \\
8-3,60 \\
10-4,71 \\
11-3,12 \\
11-4,105 \\
12-5,114 \\
13-458 \\
14-4,140 \\
16-2,105\end{array}$ & $\begin{array}{r}52.93 \\
351.69 \\
421.97 \\
486.13 \\
554.60 \\
642.11 \\
706.12 \\
708.55 \\
757.64 \\
784.08 \\
794.40 \\
819.55\end{array}$ & $\begin{array}{l}0.0 \\
0.2 \\
0.1 \\
0.3 \\
0.1 \\
0.0 \\
0.5 \\
0.0 \\
0.4 \\
0.1 \\
0.0 \\
0.1\end{array}$ & $\begin{array}{r}23.7 \\
41.7 \\
38.5 \\
40.0 \\
20.8 \\
0.1 \\
13.6 \\
27.3 \\
23.9 \\
28.1 \\
86.1 \\
2.1\end{array}$ & $\begin{array}{l}76.3 \\
58.0 \\
61.4 \\
59.6 \\
79.2 \\
99.8 \\
85.9 \\
72.7 \\
75.8 \\
71.9 \\
13.9 \\
97.9\end{array}$ & $\begin{array}{l}\text { Clay } \\
\text { Silty clay } \\
\text { Silty clay } \\
\text { Silty clay } \\
\text { Clay } \\
\text { Clay } \\
\text { Clay } \\
\text { Silty clay } \\
\text { Clay } \\
\text { Silty clay } \\
\text { Silt } \\
\text { Clay }\end{array}$ \\
\hline \multicolumn{6}{|l|}{ Hole 359} \\
\hline $2-6,110$ & 37.10 & 54.9 & 24.2 & 20.9 & Sand-silt-clay \\
\hline \multicolumn{6}{|l|}{ Hole 359A } \\
\hline$-4,30$ & 13.80 & 48.2 & 26.5 & 25.3 & Sand-silt-clay \\
\hline
\end{tabular}

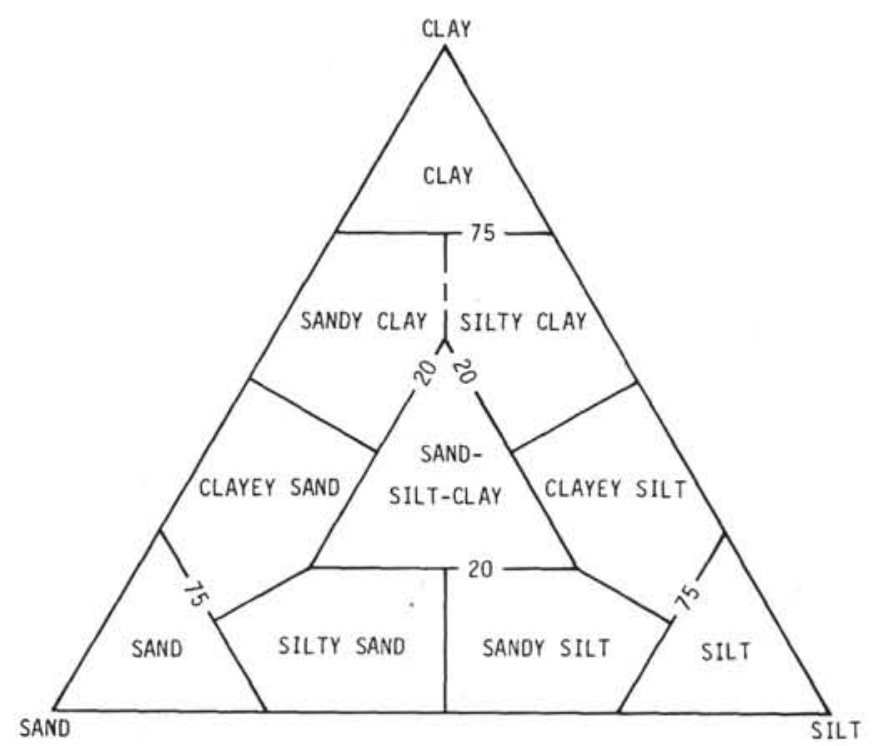

Figure 1. Sediment classification after Shepard (1954) with the sand, silt, and clay size fractions based on the Wentworth (1922) Grade Scale; Sand, silt, and clay size particles having respective diameters of 2000 to $62.5 \mu \mathrm{m}, 62.5$ to $3.91 \mu \mathrm{m}$, and less than $3.91 \mu \mathrm{m}$. Shepard's (1954) sediment classification is a function of sand, silt, and clay size percentages and not composition.

\section{CARBON-CARBONATE ANALYSIS}

Data for samples run at DSDP are listed in Table 2; these data are also presented on core forms and are included in data compilations appearing as appendices to the site report chapters (see Chapter 1 for explanation).

Leg 39 sediments were analyzed for total carbon and acid-insoluble (organic) carbon using a LECO WR-12* analyzer. The 3-cc sediment samples were dried, ground into a homogeneous powder, and redried at $105^{\circ}$ $110^{\circ} \mathrm{C}$. Two samples, a $0.1-\mathrm{g}$ and a $0.5 \mathrm{~g}$ sample, were weighed into LECO clay crucibles. The $0.1-\mathrm{g}$ sample was analyzed for total carbon without further treatment. If the sample contained less than $10 \%$ $\mathrm{CaCO}_{3}$, and additional 0.5 -g sample was analyzed for greater accuracy. The calcium carbonate percentages were calculated as follows: (\% total C - \% organic C) $\times$ $8.33=\% \mathrm{CaCO}_{3}$. Although other carbonates may be present, all acid-soluble carbon was calculated as calcium carbonate. All carbon/carbonate results are given in weight percent. For detailed step-by-step procedure and theory, see Volume 4 of the Initial Reports of the Deep Sea Drilling Project (Bader, Gerard, et al., 1970) and Volume 9 of the Initial Reports of the Deep Sea Drilling Project (Boyce, Bode, et al., 1972).

For control purposes standard sediments were made up from Deep Sea Drilling material and analyzed for total carbon at predetermined intervals with regular samples. Listed below are the data from these standards:

\begin{tabular}{|c|c|c|c|c|}
\hline $\begin{array}{c}\text { DSDP } \\
\text { Standard }\end{array}$ & $\begin{array}{c}\text { No. of } \\
\text { Samples }\end{array}$ & $\begin{array}{c}\text { Total } \\
\text { Carbon } \\
\text { as \% of } \\
\mathrm{CaCO}_{3}\end{array}$ & $\begin{array}{c}\text { Standard } \\
\text { Deviation } \\
(\%)\end{array}$ & $\begin{array}{c}\text { Maximum } \\
\text { Range } \\
(\%)\end{array}$ \\
\hline 2 & 2 & 79.80 & 0.43 & 0.75 \\
\hline 9 & 3 & 26.57 & 0.12 & 0.17 \\
\hline
\end{tabular}

TABLE 2

Carbon and Carbonate Analyses, Leg 39

\begin{tabular}{lrrrr}
\hline $\begin{array}{c}\text { Sample } \\
\text { (Interval in cm) }\end{array}$ & $\begin{array}{c}\text { Hole } \\
\text { Depth } \\
(\mathrm{m})\end{array}$ & $\begin{array}{c}\text { Total } \\
\text { Carbon }\end{array}$ & $\begin{array}{c}\text { Organic } \\
\text { Carbon }\end{array}$ & CaCO3 \\
\hline Hole 353 & & & & \\
2-2, 115 & 121.2 & 0.8 & 0.4 & 3 \\
2-3, 99 & 122.5 & 0.9 & 0.5 & 3 \\
3-2, 110 & 263.6 & 0.2 & 0.1 & 1 \\
3-2, 125 & 263.8 & 0.1 & 0.1 & 0 \\
3-2, 144 & 263.9 & 0.7 & 0.3 & 3 \\
& & & & \\
Hole 353A & & & & \\
1-2, 51 & 2.0 & 1.0 & 0.6 & 3 \\
Site 354 & & & & \\
1-1, 86 & & & & \\
1-2, 178 & 0.9 & 2.4 & 0.2 & 18 \\
3-1, 130 & 3.3 & 3.4 & 0.3 & 27 \\
\hline
\end{tabular}


TABLE 2 - Continued

\begin{tabular}{|c|c|c|c|c|}
\hline $\begin{array}{c}\text { Sample } \\
\text { (Interval in } \mathrm{cm} \text { ) }\end{array}$ & $\begin{array}{l}\text { Hole } \\
\text { Depth } \\
\text { (m) }\end{array}$ & $\begin{array}{l}\text { Total } \\
\text { Carbon }\end{array}$ & $\begin{array}{l}\text { Organic } \\
\text { Carbon }\end{array}$ & $\mathrm{CaCO} 3$ \\
\hline \multicolumn{5}{|c|}{ Site 354 - Continued } \\
\hline $4-1,120$ & 141.7 & 7.3 & 0.1 & 60 \\
\hline $4-6,20$ & 148.2 & 3.9 & 0.1 & 32 \\
\hline $4-6,60$ & 148.6 & 8.2 & 0.1 & 68 \\
\hline $5-2,65$ & 189.7 & 5.3 & 0.1 & 43 \\
\hline $5-2,138$ & 190.4 & 8.2 & 0.1 & 67 \\
\hline $6-2,22$ & 236.7 & 8.0 & 0.1 & 66 \\
\hline $6-3,65$ & 238.7 & 4.5 & 0.1 & 36 \\
\hline $6-3,96$ & 239.0 & 9.0 & 0.1 & 74 \\
\hline $6-3,102$ & 239.0 & 9.1 & 0.1 & 75 \\
\hline $6-3,139$ & 239.4 & 4.4 & 0.1 & 36 \\
\hline $7-1,139$ & 283.9 & 8.9 & 0.1 & 73 \\
\hline $7-4,96$ & 288.0 & 9.7 & 0.1 & 80 \\
\hline $8-1,106$ & 340.6 & 9.0 & 0.1 & 74 \\
\hline $8-2,6$ & 341.1 & 2.2 & 0.2 & 17 \\
\hline $9-2,95$ & 399.0 & 6.9 & 0.1 & 57 \\
\hline $10-2,6$ & 455.6 & 5.8 & 0.3 & 46 \\
\hline $10-3,21$ & 457.2 & 9.4 & 0.1 & 77 \\
\hline $11-2,11$ & 522.1 & 9.7 & 0.1 & 80 \\
\hline $11-4,145$ & 526.5 & 11.5 & 0.1 & 95 \\
\hline $12-1,98$ & 607.0 & 7.8 & 0.1 & 64 \\
\hline $12-5,89$ & 612.9 & 6.3 & 0.2 & 51 \\
\hline $13-1,149$ & 693.0 & 7.6 & 0.1 & 62 \\
\hline $13-6,124$ & 700.2 & 7.9 & 0.1 & 65 \\
\hline $14-1,107$ & 702.1 & 8.9 & 0.1 & 73 \\
\hline $14-5,27$ & 707.3 & 6.7 & 0.1 & 55 \\
\hline $15-2,26$ & 816.3 & 7.7 & 0.1 & 63 \\
\hline $15-3,71$ & 818.2 & 7.6 & 0.2 & 62 \\
\hline $16-3,48$ & 837.0 & 8.0 & 0.1 & 66 \\
\hline $16-6,121$ & 842.2 & 5.3 & 0.1 & 43 \\
\hline $17-3,80$ & 856.3 & 7.1 & 0.1 & 58 \\
\hline $18-1,90$ & 872.9 & 6.6 & 0.2 & 54 \\
\hline $18-4,128$ & 877.8 & 4.6 & 0.3 & 36 \\
\hline \multicolumn{5}{|l|}{ Site 355} \\
\hline $1-3,70$ & 56.9 & 0.1 & 0.1 & 0 \\
\hline $2-3,70$ & 113.7 & 0.6 & 0.1 & 4 \\
\hline $2-4,70$ & 115.2 & 1.7 & 0.1 & 14 \\
\hline $2-5,70$ & 116.7 & 1.1 & 0.1 & 8 \\
\hline $2-5,110$ & 117.1 & 7.7 & 0.1 & 64 \\
\hline $3-2,50$ & 169.0 & 2.2 & 0.1 & 17 \\
\hline $3-3,110$ & 171.1 & 3.1 & 0.2 & 24 \\
\hline $3-5,90$ & 173.9 & 0.1 & 0.1 & 0 \\
\hline $4-3,140$ & 218.9 & 0.1 & 0.1 & 0 \\
\hline $5-1,10$ & 423.5 & 0.2 & 0.1 & 0 \\
\hline $5-5,90$ & 250.3 & 0.1 & 0.1 & 0 \\
\hline $6-2,20$ & 264.1 & 0.3 & 0.2 & 1 \\
\hline $7-2,60$ & 283.1 & 1.4 & 0.1 & 11 \\
\hline $7-3,75$ & 284.8 & 0.1 & 0.1 & 0 \\
\hline $8-2,145$ & 303.0 & 0.1 & 0.1 & 0 \\
\hline $9-3,7$ & 322.1 & 0.1 & 0.1 & 0 \\
\hline $9-5,110$ & 326.1 & 0.2 & 0.1 & 1 \\
\hline \multicolumn{5}{|l|}{ Site 355} \\
\hline $11-3,42$ & 350.9 & 0.1 & 0.1 & 0 \\
\hline $12-4,102$ & 363.5 & 0.1 & 0.1 & 0 \\
\hline $13-2,70$ & 368.7 & 0.1 & 0.1 & 0 \\
\hline $14-5,140$ & 383.4 & 0.1 & 0.1 & 0 \\
\hline $15-1,98$ & 386.5 & 0.1 & 0.1 & 0 \\
\hline $15-1,130$ & 386.8 & 0.1 & 0.1 & 0 \\
\hline $17-2,9$ & 406.1 & 1.8 & 0.0 & 15 \\
\hline $17-2,37$ & 406.4 & 5.2 & 0.0 & 43 \\
\hline $17-3,146$ & 409.0 & 9.7 & 0.0 & 80 \\
\hline $17-4,25$ & 409.3 & 9.1 & 0.0 & 76 \\
\hline $18-1,32$ & 414.3 & 10.0 & 0.0 & 83 \\
\hline $18-2,65$ & 416.2 & 10.0 & 0.0 & 83 \\
\hline $18-3,120$ & 418.2 & 6.1 & 0.0 & 50 \\
\hline
\end{tabular}

TABLE 2 - Continued

\begin{tabular}{|c|c|c|c|c|}
\hline $\begin{array}{c}\text { Sample } \\
\text { (Interval in } \mathrm{cm} \text { ) }\end{array}$ & $\begin{array}{l}\text { Hole } \\
\text { Depth } \\
\text { (m) }\end{array}$ & $\begin{array}{l}\text { Total } \\
\text { Carbon }\end{array}$ & $\begin{array}{l}\text { Organic } \\
\text { Carbon }\end{array}$ & $\mathrm{CaCO}$ \\
\hline \multicolumn{5}{|c|}{ Site 355 - Continued } \\
\hline $\begin{array}{l}19-2,88 \\
19-3,70 \\
20-2,18\end{array}$ & $\begin{array}{l}425.9 \\
427.2 \\
434.7\end{array}$ & $\begin{array}{r}11.2 \\
9.7 \\
10.2\end{array}$ & $\begin{array}{l}0.0 \\
0.0 \\
0.0\end{array}$ & $\begin{array}{l}93 \\
81 \\
84\end{array}$ \\
\hline
\end{tabular}

Hole 356

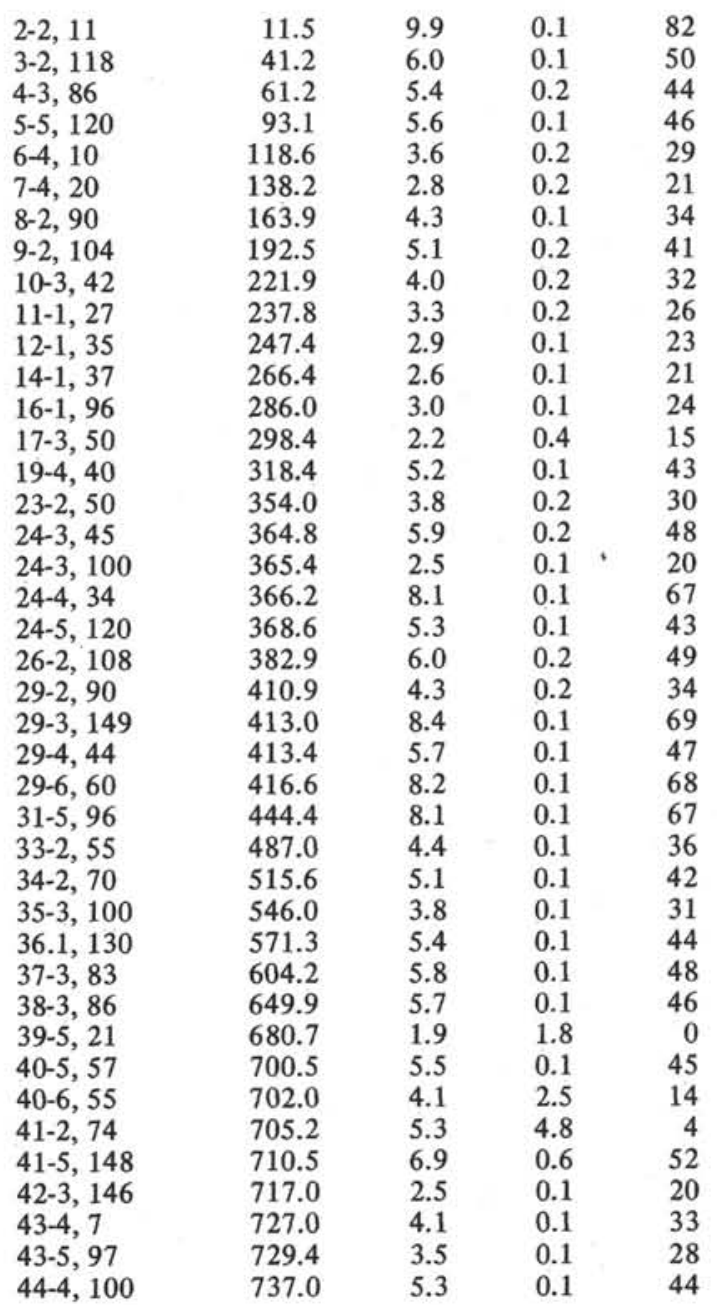

Hole 356A

$\begin{array}{lllll}1-4,80 & 24.7 & 4.0 & 0.1 & 32 \\ 1-5,95 & 26.3 & 5.1 & 0.1 & 42 \\ 2-5,12 & 34.6 & 5.6 & 0.1 & 45\end{array}$

Site 357

\begin{tabular}{lrrrr}
$1-2.100$ & 2.5 & 10.7 & 0.1 & 89 \\
$3-3,100$ & 22.0 & 10.8 & 0.0 & 90 \\
$4-6,90$ & 35.9 & 9.6 & 0.0 & 79 \\
$5-5,70$ & 43.7 & 9.9 & 0.1 & 82 \\
$6-6,41$ & 54.4 & 9.4 & 0.0 & 78 \\
$8-4,82$ & 70.8 & 10.4 & 0.0 & 87 \\
$9-4,60$ & 80.5 & 10.6 & 0.1 & 88 \\
$10-1,110$ & 95.1 & 10.4 & 0.0 & 86 \\
$11-2,94$ & 105.9 & 10.1 & 0.0 & 84 \\
$14-1,100$ & 152.0 & 9.1 & 0.1 & 75 \\
$17-3,80$ & 192.8 & 9.3 & 0.1 & 77 \\
$18-2,86$ & 210.4 & 10.0 & 0.1 & 83 \\
\hline
\end{tabular}


TABLE 2 - Continued

\begin{tabular}{|c|c|c|c|c|}
\hline $\begin{array}{c}\text { Sample } \\
\text { (Interval in } \mathrm{cm} \text { ) }\end{array}$ & $\begin{array}{l}\text { Hole } \\
\text { Depth } \\
\text { (m) }\end{array}$ & $\begin{array}{c}\text { Total } \\
\text { Carbon }\end{array}$ & $\begin{array}{l}\text { Organic } \\
\text { Carbon }\end{array}$ & $\mathrm{CaCO} 3$ \\
\hline \multicolumn{5}{|c|}{ Site 357 - Continued } \\
\hline $19-2,119$ & 239.2 & 9.4 & 0.1 & 78 \\
\hline $20-3,131$ & 259.8 & 9.9 & 0.0 & 82 \\
\hline $22-4,90$ & 308.4 & 9.3 & 0.1 & 77 \\
\hline $24-1,148$ & 352.0 & 10.4 & 0.1 & 86 \\
\hline $26-6,34$ & 387.2 & 7.7 & 0.1 & 64 \\
\hline $27-4,96$ & 413.5 & 4.0 & 0.1 & 33 \\
\hline $28-3,124$ & 440.7 & 9.2 & 0.1 & 76 \\
\hline $31-2,125$ & 495.8 & 9.1 & 0.1 & 75 \\
\hline $31-3,95$ & 497.0 & 9.2 & 0.0 & 76 \\
\hline $33-5,94$ & 528.4 & 8.4 & 0.1 & 69 \\
\hline $34-4,37$ & 555.3 & 8.4 & 0.1 & 70 \\
\hline $34-6,10$ & 558.0 & 5.7 & 0.1 & 47 \\
\hline $35-2,33$ & 580.3 & 8.0 & 0.1 & 66 \\
\hline $36-1,70$ & 607.7 & 5.4 & 0.1 & 44 \\
\hline $36-5,24$ & 613.2 & 9.0 & 0.1 & 75 \\
\hline $40-3,106$ & 696.6 & 5.0 & 0.1 & 41 \\
\hline $41-4,17$ & 707.7 & 5.3 & 0.1 & 44 \\
\hline $42-2,32$ & 713.3 & 4.9 & 0.2 & 39 \\
\hline $43-2,4$ & 723.0 & 7.0 & 0.1 & 57 \\
\hline $44-2,24$ & 732.2 & 7.2 & 0.2 & 58 \\
\hline $46-3,30$ & 746.3 & 7.3 & 0.1 & 60 \\
\hline $47-2,13$ & 751.6 & 3.8 & 0.1 & 31 \\
\hline $48-2,18$ & 760.7 & 8.3 & 0.1 & 68 \\
\hline $50-6,5$ & 785.9 & 4.6 & 0.1 & 37 \\
\hline $51-6,78$ & 795.8 & 6.6 & 0.2 & 53 \\
\hline \multicolumn{5}{|l|}{ Site 358} \\
\hline $1-5,78$ & 54.7 & 0.2 & 0.2 & 0 \\
\hline $2-2,40$ & 125.4 & 0.2 & 0.2 & 0 \\
\hline $3-2,93$ & 202.4 & 0.3 & 0.3 & 0 \\
\hline $3-5,90$ & 206.9 & 0.1 & 0.1 & 0 \\
\hline $4-1,112$ & 276.6 & 0.3 & 0.2 & 0 \\
\hline $5-2,37$ & 353.4 & 0.3 & 0.1 & 1 \\
\hline $6-1,19$ & 418.2 & 0.2 & 0.2 & 0 \\
\hline $7-1,130$ & 485.8 & 0.2 & 0.2 & 0 \\
\hline $8-2,26$ & 552.8 & 0.7 & 0.2 & 4 \\
\hline $9-2,64$ & 591.1 & 0.2 & 0.1 & 0 \\
\hline $10-4,70$ & 642.1 & 0.2 & 0.2 & 0 \\
\hline $11-2,66$ & 705.2 & 0.1 & 0.1 & 0 \\
\hline $11-4,5$ & 707.6 & 5.8 & 0.1 & 48 \\
\hline $12-3,80$ & 754.3 & 8.3 & 0.0 & 69 \\
\hline $12-5,114$ & 757.6 & 4.5 & 0.1 & 37 \\
\hline $13-1,136$ & 780.4 & 3.6 & 0.1 & 30 \\
\hline $14-1,22$ & 788.7 & 6.2 & 0.1 & 51 \\
\hline $14-4,140$ & 794.4 & 5.7 & 0.1 & 47 \\
\hline $15-1,70$ & 798.7 & 0.1 & 0.0 & 0 \\
\hline
\end{tabular}

TABLE 2 - Continued

\begin{tabular}{ccccc}
\hline $\begin{array}{c}\text { Sample } \\
\text { (Interval in cm) }\end{array}$ & $\begin{array}{c}\text { Hole } \\
\text { Depth } \\
(\mathrm{m})\end{array}$ & $\begin{array}{c}\text { Total } \\
\text { Carbon }\end{array}$ & $\begin{array}{c}\text { Organic } \\
\text { Carbon }\end{array} \mathrm{CaCO}_{3}$ \\
\hline
\end{tabular}

Site 358 - Continued

$\begin{array}{lllll}15-2,140 & 800.9 & 2.7 & 0.0 & 22 \\ 16-2,106 & 819.6 & 2.1 & 0.0 & 17\end{array}$

Hole 359

\begin{tabular}{lrrrr}
$1-1,119$ & 1.2 & 11.4 & 0.0 & 94 \\
$2-2,100$ & 31.0 & 10.6 & 0.0 & 88 \\
$2-6,122$ & 37.2 & 8.7 & 0.0 & 72 \\
$4-2,26$ & 86.8 & 1.4 & 0.0 & 12 \\
Hole 359A & & & & \\
$1-6,120$ & 17.7 & 10.6 & 0.0 & 88 \\
$2-4,110$ & 24.1 & 11.2 & 0.0 & 93 \\
\hline
\end{tabular}

\section{REFERENCES}

Bader, R.G., Gerard, R.D., et al., 1970. Initial Reports of the Deep Sea Drilling Project, Volume 4: Washington (U.S. Government Printing Office), Appendix III.

Bode, G.W., 1974. Carbon and carbonate analyses, Leg 27, Deep Sea Drilling Project. In Heirtzler, J.R., Veevers, J.J., et al., Initial Reports of the Deep Sea Drilling Project, Volume 27: Washington (U.S. Government Printing Office), p. 499.

Boyce, R.E., 1972. Grain size analyses, Leg 9, Deep Sea Drilling Project. In Hays, J.D., et al., Initial Reports of the Deep Sea Drilling Project, Volume 9: Washington (U.S. Government Printing Office), p. 745.

Boyce, R.E. and Bode, G.W., 1972. Carbon and carbonate analyses, Leg 9, Deep Sea Drilling Project. In Hayes, J.D., et al., Initial Reports of the Deep Sea Drilling Project, Volume 9: Washington (U.S. Government Printing Office), p. 747.

Hodgman, C.D., Weast, R.C.Y., and Selby, S.M., 1960. Handbook of chemistry and physics: Cleveland (Chemical Rubber Publishing Co.)

Krumbein, W.C. and Pettijohn, F..J., 1938. Manual of sedimentary petrography; New York (Appleton-CenturyCrofts, Inc.).

Shepard, F.P., 1954. Nomenclature based on sand-silt-clay ratios: J. Sediment. Petrol., v. 24, p. 151.

Wentworth, C.K., 1922. A scale of grade and class terms for clastic sediments: J. Geol., vo. 30, p. 377. 